\title{
Philosophiques
}

\section{La longue marche à travers les institutions : de Wittgenstein à}

Marx

Allocution présidentielle de M. Kai Nielsen, $28^{\mathrm{e}}$ Congrès de

l'A.C.P., juin 1984

\section{Kai Nielsen}

Volume 13, numéro 1, printemps 1986

URI : https://id.erudit.org/iderudit/203305ar

DOI : https://doi.org/10.7202/203305ar

Aller au sommaire du numéro

Éditeur(s)

Société de philosophie du Québec

ISSN

0316-2923 (imprimé)

1492-1391 (numérique)

Découvrir la revue

Citer ce document

Nielsen, K. (1986). La longue marche à travers les institutions : de Wittgenstein à Marx : allocution présidentielle de $\mathrm{M}$. Kai Nielsen, $28^{\mathrm{e}}$ Congrès de l'A.C.P., juin 1984. Philosophiques, 13(1), 113-129. https://doi.org/10.7202/203305ar d'utilisation que vous pouvez consulter en ligne. 


\section{INTERVENTIONS}

\section{LA LONGUE MARCHE À TRAVERS LES INSTITUTIONS : DE WITTGENSTEIN A MARX}

\section{(Allocution présidentielle de M. KAI NIELSEN, 28 Congrès de l'A.C.P., juin 1984.) \\ par Kai Nielsen}

Il arrive assez rarement qu'un philosophe canadien, francophone ou anglophone, prenne la peine d'esquisser son autobiographie intellectuelle. Plus rarement encore une telle tentative manifeste-t-elle un intérêt justifiant une ample diffusion. Le dernier numéro de Pbilosophiques contenait pourtant un tel exemple: «Figures de l'empirisme», le discours de réception de M. François Duchesneau (Université de Montréal) à la Société Royale du Canada, lu le 7 décembre 1984. Nous publions dans cette livraison un second exemple, qui mérite lui aussi attention: l'allocution présidentielle de M. Kai Nielsen (Université de Calgary), lors du $28^{\mathrm{e}}$ Congrès de l'Association Canadienne de Philosophie, tenu à Guelph le 10 juin 1984.

Philosophiques n'a pas l'habitude d'offrir une tribune aux textes officiels de l'A.C.P. Ce cas-ci mérite exception pour de nombreuses raisons. Tout d'abord ce texte, exemplaire à plus d'un titre, examine les modalités et finalités actuelles de la philosophie et envisage, d'un certain point de vue, son destin même. Ensuite, il dresse, panorama à l'appui, un tableau éclairant de la philosophie anglo-saxonne des trente dernières années. Enfin, parce que rapproché du discours de $M$. Duchesneau, il permet de mettre en parallèle deux traditions intellectuelles et d'obtenir un aperçu 
partiel des constellations philosophiques respectives des domaines anglophone et francophone. Cette éclaircie sur un paysage parfois peu familier aux philosophes du Québec devrait s'avérer — du moins l'espérons-nous - utile à maints égards.

La présente traduction, autorisée par l'auteur, est issue d'une première version réalisée par M.B.K. Altmann, version que nous avons, pour les besoins de l'actuelle publication, entièrement revue et très largement remaniée. Figurent parfois entre parenthèses, en regard du texte français, certaines expressions de l'original dont il nous a paru difficile de cendre en notre langue toutes les résonnances sémantiques appropriées. Les notes appelées par un chiffre sont de l'auteur. Nous avons estimé utile d'ajouter à l'occasion certaines précisions: ces notes sont, elles, appelées par une lettre et nous les avons réduites au strict minimum afin de ne pas rompre le rythme général de l'allocution.

Nous voudrions, en terminant, remercier M. Yvan Cloutier (Collège de Sherbrooke) qui a porté à notre attention l'existence de ce texte de même que $M$. André Morazain (Collège de Rimouski) qui a émis plusieurs remarques pertinentes touchant l'esprit général de cette traduction.

Jean-Claude Simard.

1

Cette allocution sera résolument méta-philosophique. Elle reflétera à un certain degré mon attitude ambivalente à l'égard de. la philosophie. Plus encore peut-être que la majorité de ceux qui ont formé leur conception de la philosophie au cours des vingt années qui ont suivi la deuxième guerre mondiale, $j$ 'ai des doutes sur l'utilité de faire de la philosophie et sur ce qu'elle peut accomplir, et je m'interroge à propos de ce qu'elle devrait être. L'air de satisfaction béate qu'ont beaucoup de philosophes envers leur discipline me décourage et m'étonne. Ils ne se rendent pas compte apparemment à quel point notre branche de la connaissance n'a qu'une importance marginale aux yeux de beaucoup de monde (y compris l'intelligentsia) hors de son domaine ${ }^{1}$.

1. La remarque de Peter Worsley, spécialiste d'anthropologie culturelle, dans son excellent petit livre sur Marx, est typique d'une telle attitude à l'égard de la 
J'essayerai d'aborder ces problèmes et de poser quelques questions, étant donné les connaissances et la situation actuelles, touchant ce que, possiblement, la philosophie peut être, et ce à quoi elle devrait aspirer. Afin de procéder de façon rationnelle, il faut parler un peu du rôle que la philosophie a joué récemment et du succès des projets de recherche qu'elle a ențrepris.

Il est évident qu'on a affaire à un sujet vaste et tendancieux. Si on veut en discuter sans se borner exclusivement à des lieux communs, on est obligé de choisir un angle spécifique sous lequel on présentera la discussion, mais inévitablement beaucoup des aspects capitaux du sujet seront passés sous silence. $A$ mon avis, il est possible de contrebalancer ce problème en en parlant de façon autobiographique; c'est-à-dire que je ferai quelque peu état des philosophes, des méthodes et des positions philosophiques qui m'ont formé. Une telle approche serait égocentrique s'il n'y avait de bonnes chances que ma formation ait beaucoup ressemblé à celle d'un bon nombre de mes collègues de la même génération de sorte qu'on pourra vraisemblablement en tirer une leçon. Je commence donc par quelques réflexions autobiographiques.

Vers 1950, à l'époque où j'étais en train de finir ma thèse, le monde philosophique anglophone tout entier fut pénétré rapidement par un mouvement de vaste ampleur: les idées du Wittgenstein du post-Tractatus ainsi que quelque chose d'apparenté quoique plus prosaïque, à savoir ce qu'on appelait la philosophie d'Oxford ou du langage ordinaire. Ce mouvement est apparu d'abord dans la philosophie d'Oxford (surtout celle de Ryle), dans les manuscrits du Blue Book et du Brown Book, et dans deux collections d'articles de John Wisdom. (Je me souviens encore de l'allégresse que j'ai ressentie quand j'ai lu le Blue Book d'un seul trait: il me sembla alors que les écailles me tombaient des yeux). Un peu plus tard les Philosophical Investigations devenaient accessibles. On en parlait avec animation, avec amertume même, chez les professeurs plus âgés. Leurs méthodes

philosophie. Dans un passage où il parle de la philosophie du milieu du XIX ${ }^{\mathrm{e}}$ siècle par opposition à celle d'aujourd'hui, Worsley remarque, sans d'ailleurs s'y arrêter, que «Philosophy was not a rather marginal academic field of study, as it tends to be today» (La philosophie n'était pas le domaine assez marginal qu'elle tend à être présentement). Peter Worsley, Marx and Marxism (London : Tavistock Publications, 1982), p. 22. 
étaient déjà assez bien arrêtées et ces nouvelles idées leur paraissaient un défi. Quant à moi, elles ont changé ma conception et ma pratique de la philosophie. Leur influence s'est manifestée de trois façons : d'abord, elles confirmaient et - c'est ce qu'il me semblait alors - justifiaient de manière éclatante mon rejet de la métaphysique et de l'épistémologie. C'étaient les premiers intérêts que j'avais pris à la philosophie qui m'avaient prédisposé à ce refus, et les études que j'avais entreprises, d'abord du pragmatisme et ensuite de l'empirisme logique, l'avaient renforcé l'une après l'autre. Mais c'est l'étude de Wittgenstein qui me l'imprima profondément dans l'esprit.

Deuxièmement, Wittgenstein me détourna de l'étude de la logique et de ce qu'on appelait la philosophie du langage idéal. Avant l'assaut de Wittgenstein, j'avais été influencé, en rédigeant ma thèse, par C.I. Lewis et Rudolf Carnap. Bien qu'il me semblât que ce qu'il y avait de plus important dans leurs ouvrages ne devait rien à l'application des procédés logiques, leur autorité était telle que je me sentais obligé d'utiliser ces procédés malgré ma propre inclination; mais je n'arrivais absolument pas à comprendre comment leur usage m'aiderait à résoudre ou à dissoudre l'une ou l'autre des questions philosophiques qui m'intéressaient. Wittgenstein (aidé de Moore, Wisdom, Waismann et Austin) mirent fin à tout cela. Je compris comment on pouvait entreprendre quelque chose de rigoureux et de conceptuel sans se prévaloir de ces activités logiques qui me semblaient inutiles. L'on n'avait pas besoin d'un langage idéal strict; ce qu'il fallait, c'était une compréhension du fonctionnement de notre propre langage, assez claire pour apporter une réponse aux perplexités philosophiques surgissant de nos méprises sur ce fonctionnement. Nous réfléchissions assez bien avec ce langage dans des contextes courants, mais dès qu'il s'agissait de réfléchir sur ce langage, des problèmes liés à certaines questions philosophiques surgissaient qui nous laissaient perplexes. (Bien entendu, la plupart des philosophes plongés dans cette perplexité voyaient la situation différemment. C"était la tâche principale des philosophes inspirés de Wittgenstein de démontrer que ces perplexités philosophiques renvoyaient au mécanisme de leur langage).

Le troisième changement que Wittgenstein m'imposa, et peut-être le plus important, ce fut un déplacement total de mon 
travail en éthique. Quand j'ai commencé à étudier Wittgenstein, j'étais en train d'élaborer une défense de la théorie émotiviste de l'éthique contre les critiques rationalistes et même contre ceux qui y étaient moins opposés tels Hare et Nowell-Smith. J'étais d'avis et je le suis toujours, que les ouvrages de Stevenson et de Hägerström sont plus puissants qu'on ne pense d'habitude, bien qu'il m'ait semblé également, à l'époque, qu'ils avaient raison à peu de choses près. L'étude de Wittgenstein et du langage ordinaire changea tout cela. J'en vins à penser que nous devrions nous concentrer moins sur le statut logique des énoncés évaluatifs et davantage, dans les contextes spécifiques de la moralité et de la politique, sur ce qui constitue une bonne justification en éthique. Nous devrions nous inquiéter moins de ce que «bien» veut dire, et nous concentrer plutôt sur ce qui constitue de bonnes justifications et sur la structure sous-jacente au raisonnement moral. Il me semblait que ce que nous faisions, Toulmin, Findlay, Baier et moi (ainsi que Rawls d'ailleurs au tout début de sa carrière) c'était, dans le domaine de la philosophie morale, un travail analogue à celui que Wittgenstein et Wisdom avaient entrepris pour la psychologie philosophique, la philosophie de l'esprit et la philosophie du langage ${ }^{2}$. Et je m'attendais à un développement similaire dans les domaines de la philosophie politique, sociale et du droit. (Le travail de Hart donnait l'impression d'ouvrir une telle voie).

Ces jours-là sont bien éloignés maintenant, mais les idées qui m'animaient alors ont pénétré mes premiers travaux sur l'éthique et aussi, assaisonnées d'une pointe d'empirisme logique, mon travail sur la philosophie de la religion. Il faut cependant préciser que quelques-uns des aspects de ce développement me sont tout particuliers, alors que le mouvement général d'opposition à la philosophie du langage idéal et l'élaboration de procédures inspirées de Wittgenstein sont communs aux philosophes de ma génération.

2. À ce propos, voici mes articles les plus importants: "The Functions of Moral Discourse», The Philosophical Quarterly Vol. 7 (July, 1957); "Justification and Moral Reasoning», Metbodos Vol. 8, nos 33-34 (1957) ; "Good Reasons in Ethics», Theoria Vol. XXIV (1958); «Appraising Doing the Thing Done», Journal of Philosophy Vol. LVII (November, 1960) ; "The Good Reasons Approach Revisited ", Archiv für Rechts und Sozialphilosophie (1964); and «Moral Truth» in Studies in Moral Pbilosophy, édité par Nicholas Rescher (Oxford, 1968). 
Par la suite, je commençai lentement à déroger au Weltgeist philosophique. Cela s'est produit graduellement, et sans aucun dessein iconoclaste de ma part. Mais ce n'en est pas moins devenu une réalité.

Wittgenstein n'a pas étudié le langage de façon systématique. Il n'a pas essayé de bâtir une philosophie du langage, ni de se servir des résultats de la linguistique ou de contribuer à son développement, ni de combiner philosophie et linguistique. Il cherchait plutôt à nous libérer de l'emprise qu'une image erronée du langage peut exercer, en nous rappelant comment le langage fonctionne dans ces contextes particuliers où se vit une véritable perplexité philosophique. Mais cela ne nécessite pas une théorie de remplacement, même si nous devons nous entraîner à comprendre le fonctionnement de notre langage et être sensibles aux problèmes philosophiques.

Cependant, la philosophie commençait à prendre une nouvelle tournure sous les influences différentes d'Austin en Angleterre et de Quine et Chomsky en Amérique du Nord (dont les influences différaient aussi entre elles). On crut d'abord qu'Austin était un Wittgenstein plus exigeant, cherchant moins à faire école (less programmatic), moins passionnant; mais quand sa quête du performatif tourna court dans le dernier tiers de How to do Things with Words, il devint évident que l'analyse d'Austin ne se liait plus à des problèmes philosophiques identifiables mais s'intéressait plutôt au langage en tant que tel et se transférerait donc mieux à la linguistique. Même là où de nouveaux ouvrages traitaient un problème philosophique, tel Semantic Analysis de Paul Ziff, il devenait suffisamment clair, dès qu'on examinait attentivement le livre, que l'analyse du «bien» aurait pu se faire sans l'équipement linguistique complexe dont Ziff s'était servi. Je me retrouvai bientôt, à mesure que se développaient de tels travaux, très mal disposé à l'égard de ce virage linguistique des années 60 et 70 . Même aujourd'hui il me semble que ce mouvement n'a jamais incorporé ce que Wittgenstein proposait quant au rôle du langage dans le contexte des activités philosophiques. De toute façon, j'avais l'impression que c'était une mauvaise direction et je n'en ai tenu aucun compte après ses premières manifestations. 
Je cultivais de préférence mon propre jardin en continuant mon travail sur la philosophie morale, tout en m'inquiétant de moins en moins du statut logique de mes projets, et en me demandant moins fréquemment si je faisais ce que Wittgenstein aurait appelé des «remarques grammaticales», ou si je faisais de l'éthique normative ou de la méta-éthique, ou encore si les philosophes pouvaient s'occuper correctement d'éthique normative. A un moment donné, il m'avait semblé absolument essentiel de tracer ces délimitations. J'étais alors persuadé qu'il fallait circonscrire le terrain afin d'assurer des progrès dạs notre examen de la moralité et d'être sûr de ce qu'était faire de la philosophie. Mais cette conviction s'est lentement dissipée. J'ai alors essayé plutôt de penser aussi soigneusement que possible aux questions morales et, graduellement, aux questions politiques, tout en me servant d'un vocabulaire philosophique très restreint même si je continuais de prêter attention au fonctionnement de notre langage et d'établir des distinctions pertinentes au contexte lorsque cela s'avérait plus clair, ce qui était souvent le cas. (C'est une leçon que j'ai tirée de Moore, Wittgenstein et Austin, bien que Sidgwick et Broad enseignent la même chose; ce dernier a cependant tendance à le faire à l'excès et de façon pédante).

Peu après, ce fut l'entrée en scène de Quine, suivi de Sellars et Davidson. Ma prédilection pour une philosophie du langage ordinaire et ma conviction que Wittgenstein avait porté le coup fatal à toute philosophie du langage idéal m'avaient rendu, je le regrette à présent, complètement insensible à leurs méthodes. Jusqu'au milieu des années 70 , je considérai leur travail comme une suite de démarches réactionnaires (philosophiquement parlant) qui nous ramenaient en arrière, faisant totalement fi et des énormes progrès que Wittgenstein avait apportés au domaine et de plusieurs des acquis qui me semblaient révolutionnaires chez les empiristes logiques. Avec son usage de la logique, Quine ne faisait que brouiller les idées ou les traiter avec pédantisme. (Metbods of Logic, c'était une chose, Word and Object en était une tout autre). Et - du moins le pensais-je - Grice et Strawson avaient tout à fait détruit la sottise qui consiste à ne pas chercher l'établissement d'une distinction significative entre l'analytique et le synthétique. 
C'est ainsi que j'avais l'impression, à ce moment, que la philosophie analytique des années 60 et 70 avait rétrogradé et avait complètement perdu de vue les progrès de la révolution menée par Wittgenstein. On abordait l'étude de l'histoire de la philosophie de la même façon qu'auparavant, sauf qu'on se servait maintenant plus fréquemment des procédés logiques pour expliquer des textes classiques. Il y avait une préoccupation étendue pour la philosophie de la logique et du langage tandis que la philosophie de la philosophie, terrain de Wittgenstein, était tout à fait abandonnée; à la place, nous nous interrogions sur le sens du mot «usage» dans Wittgenstein, ou bien nous traitions de manière scolastique l'argument du langage privé. Nous étions assez nombreux à croire que la discussion de l'usage avait aussi peu d'intérêt que celle du sens. Il se peut que l'importance particulière accordée à la philosophie de la logique et à la philosophie du langage nous ait aidés à comprendre la logique, le langage et (si une telle chose existe) les fondements des mathématiques. Mais elle nous a très peu aidés à réfléchir clairement aux problèmes spécifiquement philosophiques. La philosophie des sciences (en tant qu'elle se distingue de l'histoire des sciences) était impliquée de la même façon et était aussi moribonde. Seule la philosophie de l'esprit semblait progresser, quoique même dans ce domaine, on trouvât des gens pour la rendre obscure et l'amoindrir, à seule fin de répondre au goût de beaucoup de philosophes pour les casse-tête. (En fait, on dirait qu'elle se réduit à cela pour certains philosophes).

Les courants dominants de la philosophie morale et de la philosophie sociale étaient eux aussi agonisants, se permettant souvent d'opérer des distinctions sans but apparent et se limitant souvent à singer dans le domaine de la morale une philosophie du langage qui avait autrefois été à la mode. (La méta-éthique employait la philosophie du langage surannée de la décennie précédente).

À l'aube des années 60, alors que j'étais également en train d'étudier l'ouvrage Thought and Action de Stuart Hampshire, je me suis mis à examiner le travail des principaux philosophes continentaux. Tout en admirant vivement depuis cette époque plusieurs des positions politiques de Sartre et de Merleau-Ponty, et tout en appréciant de temps en temps leurs écrits politiques, 
leurs grands travaux (ce que Hare a appelé leurs «big books») m'ont toujours paru impénétrables et pleins de ce non-sens métaphysique que les empiristes logiques nous ont à juste titre appris à abandonner. (Peut-être existe-t-il également une métaphysique utile, mais le type dont eux se servaient ne l'est certainement pas). Je suis prêt à croire qu'on pourrait, faisant preuve de patience, dé-mythologiser leur travail. Qui plus est, j'ai le sentiment que ce qu'ils ont entrepris est en fait d'une importance certaine, surtout si on le compare avec les visées de ceux qui s'efforcent uniquement à résoudre des casse-tête; mais l'obscurité et l'indiscipline de leur travail sont si épouvantables que, pour ma part, je n'ai aucune envie de m'attaquer à une telle épuration.

\section{III}

Deux événements qui ont eu lieu pendant les années 60 ont beaucoup influencé la philosophie. Le premier, extérieur au domaine, fut la guerre du Vietnam; et le second, lié au développement de la philosophie, fut la publication en 1970 du livre $A$ Theory of Justice de John Rawls. La guerre, et surtout la réaction des étudiants face à ce conflit, a obligé les philosophes à réfléchir plus sérieusement à la moralité politique et aux problèmes sociaux et politiques. C'était évident aux États-Unis en particulier mais cela se manifestait également en Europe et ici.

Nous nous sommes rendus compte tout de suite que nous n'étions pas prêts à aborder ces questions. La philosophie politique était le domaine le plus sous-développé de la philosophie analytique. En outre, la philosophie morale, son bras droit, s'était tellement limitée à l'aspect méta-éthique que les philosophes trouvaient bien peu à dire, malgré leur évidente bonne volonté. On devait reconstruire même ces analyses sociales décousues que nous avons l'habitude de rencontrer dans Inquiry, dans Philosophy and Public Affairs et dans Ethics. Nous sommes parvenus quelquefois à énoncer des idées dignes d'attention mais nous ne savions pas faire ce que nos collègues européens avaient appris : nous n'arrivions pas à unifier ces fragments d'analyse dans un cadre qui leur eût donné une signification plus large et qui nous eût permis par conséquent d'avoir une compréhension plus étendue de la vie sociale et de la politique. 
Le livre de John Rawls, A theory of Justice, a redonné à la philosophie anglophone un type de philosophie socio-politique systématique et totalisante (a). C'était aussi, en anglais, et pour la période d'après-guerre, le premier ouvrage de pensée morale et politique qui éveillât beaucoup d'intérêt en dehors des cercles philosophiques. De plus, il nous indiquait comment faire de la philosophie morale et sociale de façon systématiquement disciplinée et sophistiquée, et comment, ce faisant, nous pourrions éviter des questions renvoyant à la méta-éthique ou à l'analyse de concepts moraux. Afin de nous attaquer à une théorie sociale et morale, même à un niveau très fondamental, nous n'étions pas obligés de répondre à des questions sur le statut logique des énoncés moraux, de déterminer le sens des termes «morale» ou même « justice», de savoir si nous pouvions tirer d'un «est» un «devoir-être» ou si et comment des énoncés moraux étaient vrais ou faux. Rawls nous a également donné un très séduisant modèle consensuel de justification, grâce à sa fiction d'une position originale et à son appel à des jugements pondérés faisant preuve d'un profond équilibre réflexif, modèle qui survivra à l'échec du fondationalisme. Cette façon de procéder survivra aussi au rejet de ses principes particuliers de justice, au fait - il faut bien l'admettre - qu'un point archimédien tel qu'il l'a cherché ne saurait être découvert, et à cette prise de conscience: la méthode contractuelle souffre d'un préjugé libéral et d'une sociologie politique peu réaliste.

J'ai critiqué Rawls sur plusieurs aspects et à plusieurs reprises, mais il me semble qu'il nous a montré comment la philosophie et même la philosophie sociale peuvent aller de l'avant ${ }^{3}$. Il a dépassé de très loin l'analyse linguistique orthodoxe que pratiquaient Hare, Stevenson et Foot, mais aussi l'approche des bonnes justifications qui a toujours eu du mal à transcender une morale purement descriptive.

Selon Richard Rorty, le travail de Rawls n'a rien de spécifiquement philosophique, même s'il constitue une entreprise

(a) «holistic». Il n'existe pas, à notre connaissance, d'équivalent exact de ce terme en français. Selon le contexte des nombreuses occurrences, nous l'avons rendu par «totalisant", " global» ou " englobant».

3. Kai Nielsen, Equality and Liberty: A Defense of Radical Egalitarianism. (Totowa, New Jersey : Rowman and Allenheld, 1984), chapitres 2-5. 
considérable. Il aurait pu aussi bien être écrit par un politicologue, un économiste, un avocat ou quelqu' un d'apparenté. Il me semble que Rorty exagère, mais il y a malgré tout du vrai dans une telle évaluation. Pour écrire $A$ Theory of Justice, le spécialiste d'économie ou de science politique aurait dû avoir une connaissance approfondie de l'histoire de la pensée morale et sociale. De plus, on trouve dans l'œuvre de Rawls des traces du rejet par Quine de toute interprétation significative de la distinction entre analytique et synthétique, la marque du modèle de justification consensuel et anti-fondationaliste de Goodman et une compréhension raffinée du sort de la preuve dans le domaine de la philosophie. Son ouvrage trahit un type de compétence que, vraisemblablement, seul un philosophe peut manifester. Un économiste ou un politicologue n'a habituellement pas ces conreptions à l'esprit, à moins d'avoir reçu une formation philosophique approfondie ; cela n'empêche pas cependant qu'une analogie très poussée ne puisse être implicite dans ses procédés, de sorte que le traité qu'il écrirait pourrait ressembler beaucoup, en terme de contenu, à celui de Rawls. C'est-à-dire qu'un spécialiste d'économie ou de science politique pourrait bien écrire une œuvre de structure semblable à celle du livre de Rawls, sans aucune autre connaissançe de la philosophie que celle de l'histoire de la théorie morale.

Mais c'est précisément cela qui sous-tend l'exagération de Rorty. Rawls n'établit aucune distinction analytique/synthétique, distinction qui aurait pour effet de reléguer la philosophie et la théorie morale dans le domaine de l'analyse conceptuelle; il ne réfère à aucune connaissance spécialement philosophique (quoi que cela puisse signifier) et ne fait appel à aucun de ces outils analytiques que l'on dit très puissants, contrairement à ce qu'auraient tout naturellement fait Reichenbach, Quine et Dummett. Il n'y a rien dans le travail de Rawls, que ce soit au niveau de la structure très substantielle de l'ouvrage ou au niveau de la méthodologie, qui soit spécifiquement philosophique. En fait, la plus grande partie du texte aurait pu venir de quelqu'un muni d'une formation comparable à celle de Marx, Weber ou Durkheim, plutôt qu'à celle de R.M. Hare, Peter Geach, John Searle, Gilbert Hartman ou Philipa Foot. (Les aspects inspirés de Quine et Goodman dont on a fait mention ci-dessus ne sont pas essentiellement philosophiques non plus). 
À mon avis, la voie de Rawls, de même que la défaite des interprétations substantielles de la distinction analytique/synthétique, le refus de tout appel au donné, l'ébranlement des théories du vrai comme correspondance qui veulent dépasser le minimalisme de Tarski et, enfin, la reconnaissance des assertions inattaquables des historicistes touchant la connaissance, donnent à la philosophie, qui se distingue moins nettement maintenant des sciences humaines, la possibilité de nouvelles directions. (Je reviendrai sur cela dans un instant). Mais nous voyons encore une fois jusqu'à quel point la destruction du fondationalisme en philosophie est profonde et englobante.

Je n'ai pas une très haute opinion des tournants positifs de Richard Rorty en philosophie ${ }^{(b)}$ et il me semble que son travail sur l'herméneutique et le vrai est plutôt baclé. Mais je suis d'accord en général avec ses remarques touchant l'importance centrale de Wittgenstein et Dewey et avec son observation selon laquelle Quine et Sellars nous ont conduits à des résultats comparables en restant à l'intérieur de la philosophie analytique traditionnelle. Cette opinion colorait mes remarques dans le paragraphe précédent. L'essentiel de la critique de Rorty aboutit à miner tout l'édifice classique de l'épistémologie, à repousser tout fondationalisme ou tout essentialisme, même non trivial, à rejeter toute croyance en une entreprise qui pourrait porter avec quelque vraisemblance un titre tel « les fondements conceptuels» de ceci ou de cela, ou à bannir toute analyse conceptuelle qui pourrait prétendre à un résultat tel qu'on obtiendrait certaines catégories philosophiques bien définies, lesquelles jouiraient d'une place privilégiée dans la critique de la culture, y compris les catégories visant l'analyse de la science, du droit, de la moralité ou du sens commun. L'analyse conceptuelle non plus que l'analyse linguistique ne peuvent accoucher de résultats similaires à ceux dont se croyait capable la philosophie analytique classique, et la critique de la culture que les diverses traditions de la philosophia

(b) «Richard Rorty's positive turns in philosophy». Allusion vraisemblablement à l'une des thèses de Consequences of Pragmatism (Minneapolis, University of Minnesota Press, 1982). Habituellement négligées dans le monde anglo-saxon, certaines philosophies, dont le pragmatisme classique américain, ont contribué de manière authentique (Rorty parle de "positive contributions») à la position du problème actuel de la philosophie. Il faut récupérer ces aspects positifs. 
perennis se croyaient en mesure de réaliser a été elle aussi définitivement écartée. Ce sont Wittgenstein, Dewey et (selon Rorty) Heidegger qui ont effectué ce tournant. Il est peu surprenant que nous ayons mis si longtemps à nous rendre compte d'une révolution intellectuelle d'une telle ampleur. Après tout, nous nous sentons menacés par de pareils changements et nous dressons aussitôt des mécanismes de défense. Mais je crois que Rorty a correctement rendu compte de ce qu'a accompli Wittgenstein par ses traits rapides, courageux et ironiques (c'est ce que nous en pensions intuitivement après une première lecture), et de ce que Dewey avait fait plus tôt tout autrement et dans un langage très différent. (C'était peut-être l'effet profond que Dewey m'avait fait pendant mes premières années universitaires et la façon dont cet effet avait survécu à l'impact de l'empirisme logique et de l'analyse linguistique, qui m'avaient laissé si réceptif au travail de Wittgenstein).

Mais quand Rorty prend son tournant positif, il devient trop ampoulé. Il fait alors montre d'une attitude désinvolte. Cette attitude est appropriée lorsqu'il s'agit de discuter la façon dont nous devrions structurer les universités dans notre contexte culturel ; mais comme procédure touchant le type de transformation de l'humanité que doit viser la philosophie, c'est une démission. Mais alors que pouvons-nous faire après les échecs de l'empirisme logique et de l'analyse linguistique doctrinaire (programmatic)? Peut-il y avoir à présent une renaissance philosophique telle que la souhaitait Dewey? (c) Peut-être que non; peut-être la philosophie est-elle condamnée à devenir une discipline de plus en plus marginale, à mesure que se poursuit le désenchantement du monde (de-mystification of the world). Il se pourrait qu'elle soit réduite au statut d'une spécialité très étroite. Si quelqu'un voulait se renseigner au sujet de la logique déontique des désignateurs rigides, la philosophie pourrait lui fournir des réponses. Mais si on ne peut pas intégrer ces considérations dans un contexte plus large, elle restera exclusivement entre les mains des spécialistes.

(c) Allusion sans doute au programme proposé par Dewey dans son ouvrage immédiatement postérieur à la Première Guerre Mondiale, Reconstruction in Philosophy. 
Mais peut-être la philosophie n'est-elle pas complètement vouée à ne devenir qu'une petite spécialité de quelques individus. (Vous, vous faites des greffes de rein, moi je fais de la logique déontique). Rappelons le mot de Dewey selon qui la philosophie recouvre ses forces quand les problèmes de l'homme deviennent les problèmes du philosophe; et Rawls, évitant les procédés de l'analyse linguistique, s'est servi de toutes sortes d'affirmations empiriques (y compris de quelques-unes puisées dans les sciences humaines) pour bâtir, de façon assez rigoureuse, une théorie morale englobante qui, non seulement, caractérisait nos capacités morales mais qui, systématiquement, avançait des énoncés normatifs liés étroitement à la théorie sociale.

Malgré cela, l'on se rend compte que la théorie de Rawls se limite trop à la description de nos capacités morales; ce qu'il nous faut, c'est quelque chose qui dépasse Rawls sans se transformer en obscurantisme ou en idéologie. Nous voudrions, si possible, une théorie morale et sociale qui pourrait entreprendre une critique de la culture, de la société et de l'idéologie. En outre, elle devrait en être le compte rendu descriptif, explicatif et interprétatif. Elle devrait nous permettre de mieux comprendre ce que nous sommes, ce que nous avons été et ce que nous pourrions devenir. Mais elle devrait avoir aussi une visée émancipatrice et critique. Elle devrait nous indiquer comment arriver à nous améliorer et comment la société pourrait être non seulement plus juste (car la justice n'est pas le seul critère d'une évaluation de la société) mais aussi, réellement plus humaine. De plus, une telle théorie devrait nous aider à mieux comprendre les moyens par lesquels on atteint ces buts. Elle ne devrait pas se contenter de décrire l'émancipation de l'être humain; elle devrait fonctionner comme la théorie naissante d'une telle société, comme quelque chose qui décrit, même en termes généraux, l'allure qu'aurait cette société, comment elle pourrait émerger et se maintenir. La théorie aurait à justifier aussi la prétention qu'une telle société est véritablement meilleure que celle que nous connaissons et même, qu'elle mérite effectivement d'être appelée humaine. Il est possible que les empiristes logiques et les tenants de la philosophie analytique aient eu raison de croire qu'on ne pourrait jamais élaborer une théorie de ce genre. Pas plus que tout autre domaine qui se veut raisonnable, la philosophie ne devrait viser si haut. 
Les aspirations de Rawls ne sont certainement pas si ambitieuses. Il offre un tableau de nos capacités morales et propose une justification du libéralisme et de nos démocraties traditionnelles mais il ne va pas jusqu'à critiquer la culture ou élaborer une défensive tous azimuts contre les alternatives à une société libérale. Il donne une explication du capitalisme en vigueur dans l'État-Providence libéral, mais il ne démontre pas sa supériorité face au marxisme ou à l'anarchisme ou même face à l'élitisme réfléchi et aux sociétés qui accompagnent ces conceptions. Ce que nous aurions aimé avoir ici, c'est une évaluation de ce qu'on pourrait en dire d'une façon ou d'une autre.

Si Rorty est raisonnablement justifié de critiquer l'image basée sur l'épistémologie que le philosophe se fait de lui-même, alors nous ne pouvons pas l'obtenir de la théorie sociale d'un fondationaliste. La poursuite fondationaliste de l'épistémologie morale ne nous avancera pas plus que la voie d'une épistémologie plus générale. Nous ne pouvons pas emprunter la voie de Price, Hume ou Hägerström.

Je crois cependant qu'il y a une autre route qui serait peutêtre plus rentable, si on s'y engageait. C'est une route dont Rorty ne tient aucun compte, malgré l'attention qu'il apporte d'habitude à toutes les alternatives. Je la formulerai indirectement. $A$ la fin du $\mathrm{XIX}^{\mathrm{e}}$ siècle comme de nos jours, les grands travaux à visée synthétique et interprétative que menaient autrefois des philosophes tels que Hobbes, Locke, Smith, Condorcet, Hume, Kant et Hegel ont été entrepris au bout du compte par des spécialistes des sciences humaines englobantes tels que Marx, Weber et Durkheim. Ces derniers nous donnent ce que nous trouvions jadis dans Leviathan. Ils font de façon totalisante une analyse interprétative, explicative et critique de la société qui est, en dépit de la position wertfrei de Max Weber qui affirme le contraire, descriptive, explicative et, de façon critique, normative.

Nous ne savons pas encore comment nous y prendre dans le contexte d'une théorie globale. Il faut construire le bateau tout en étant à bord, puisqu'en élaborant de telles théories, il faut évaluer de manière critique ce que nous sommes en train d'entreprendre, ou du moins $\mathrm{y}$ réfléchir. Les sciences humaines sont farcies d'obscurités, d'idéologies déformantes et même, souvent, d'apologies idéologiques qui peuvent se présenter ouvertement et 
délibérément mais qui, plus fréquemment et plus insidieusement, s'y trouvent de façon involontaire. Pourtant ce sont ces sciences qui nous renseignent, plus que toute autre chose, sur ce que nous sommes, ce que nous avons été et ce que nous pourrions vraisemblablement devenir.

L'essentiel, c'est de comprendre qu'on est ici en présence de quelque chose qui enjambe la division habituelle des disciplines, en particulier la philosophie et la sociologie. Entre autres choses, il y a place ici pour la conception traditionnelle du travail analytique en philosophie, car les théories importantes d'autrefois ont quelquefois besoin, en même temps ou avant qu'on élabore de nouvelles théories critiques pour remplacer les anciennes, de ce qui est fait avec éclat par G.A. Cohen pour Marx et par Charles Taylor pour Hegel ${ }^{4}$. C'est-à-dire que nous devons reformuler de façon raisonnée les œuvres historiquement influentes et importantes qui contiennent vraisemblablement plus qu'une parcelle de vérité. Un tel travail présenterait une version mieux ordonnée de l'original et comprendrait légitimement des clarifications de son style classique: procédés destinés à établir des distinctions pertinentes en relevant les ambiguïtés et les possibilités non exprimées, reformulations plus claires et autres choses du genre. Broad, Wittgenstein et Austin en donnent l'exemple, et cela revient avant tout à être très conscient du fonctionnement de notre langage. Sur ce point, Rorty a parfaitement raison d'affirmer, s'opposant en cela à Reichenbach, que nous ne devons pas prétendre avoir des instruments analytiques puissants, ou être sur le point de nous en procurer ou d'en avoir besoin '. Ce qu'il nous faut, c'est une bonne compréhension de la théorie que nous retravaillons et des alternatives à cette théorie de même qu'une excellente capacité à établir des distinctions relevant du langage naturel. Ce n'est pas sans raison que je cite des exemples. Les procédés de Cohen dans son analyse et sa critique de Marx sont

4. G.A. Cohen, Karl Marx's Theory of History (Oxford, England: Oxford University Press, 1978), Charles Taylor, Hegel (Cambridge, England: Cambridge University Press, 1975) et Charles Taylor, Hegel and Modern Society (Cambridge, England: Cambridge University Press, 1979).

5. Richard Rorty, Consequences of Pragmatism (Minneapolis, Minnesota : University of Minnesota Press, 1982), pp. 211-230. 
très révélateurs. Son travail est une brillante application de ce dont je viens de parler ${ }^{6}$.

Cependant la philosophie, abordée du point de vue que je préconise, veut aller plus loin, en direction d'une théorie critique systématique. Mais où cela mènera n'est pas clair. Je crois, m'appuyant ici sur Quine plutôt que sur Wittgenstein, que nous devons, en utilisant ce qu'il y a à notre disposition et en essayant d'être aussi clairs que possible, progresser sur la voie d'une théorie sociale globale, en lien constant avec une pratique présumément émancipatrice, sans trop nous demander si ce que nous faisons est de la philosophie, de la sociologie, de l'économie, de l'histoire sociale, de la critique sociale ou autre chose encore. L'important c'est de nous préoccuper de la véracité, des justifications et de l'importance de nos dires, et de la façon dont ils correspondent aux autres choses que nous affirmons, et ainsi de suite. Mais ne nous demandons pas si c'est de la philosophie et ne nous demandons pas, comme Wittgenstein le fait dans la dernière partie des Investigations, si nos remarques sont "grammaticales» et donc proprement philosophiques. Demandons-nous plutôt si elles sont raisonnablement claires, s'il y a de bonnes raisons de les croire et, étant donné leur présumée véracité, si elles sont importantes - surtout pour nous donner prise sur ce que nous sommes, ce que nous avons été et ce que nous pourrions devenir. Si nous pouvons accomplir cette tâche de façon relativement rigoureuse, nous aurons sans conteste accompli quelque chose d'utile. Qu'ensuite nous décidions ou non de l'appeler philosophie n'a aucune importance. Si ce n'est pas de la philosophie, c'est quelque chose de plus important qui devrait la remplacer dans son traditionnel rôle culturel. Mais je vois mal pourquoi on ne pourrait pas dire que c'est un élément central de la philosophie: c'est ce que la philosophie devrait être une fois qu'elle sera reconstruite.

Université de Calgary.

6. G.A. Cohen, «Reply to Four Critics», Analyse \& Kritik Vol. 5, nº 2 (December, 1983), pp. 195-221. 